Bull. Austral. Math. Soc.

VOL. 65 (2002) [329-335]

\title{
FREE SURFACE WAVES OVER A DEPRESSION
}

\author{
J.W. CHOI
}

\begin{abstract}
Steady waves at the free surface of an incompressible fluid passing over a depression are considered. By studying a $\mathrm{KdV}$ equation with negative forcing term, new types of solutions are discovered numerically and a new cut-off value of the Froude number, above which unsymmetric solitary-wave-like wave solutions exist, is also found.
\end{abstract}

\section{INTRODUCTION}

The purpose of this paper is to study steady surface waves on a two dimensional incompressible and invisid fluid flow passing over a small depression on a flat bottom. We assume that the depth $H^{*}$ and the speed $c$ of the fluid flow far upstream are constants and an upstream Froude number $F$ is defined by $F=c /(g H)^{1 / 2}$. Steady solutions of one layer fluid for a positive obstruction have been studied numerically by $\mathrm{Wu}$ and $\mathrm{Wu}[\mathbf{9}]$, Forbes and Schwartz [3], Vanden-Broeck [8], Forbes [2], and others and asymptotically by Cole [1], Shen et al [7], S.P Shen and M.C Shen [6], Gong and Shen [4] and others. It was found in these papers that there exist a cut-off value of Froude number , $F_{1}>1$, above which two supercritical stationary solitary-wave-like waves appear and there is a critical value of Froude number, $F_{2}<1$ at which a hydraulic fall solution connecting an upstream subcritical flow to a downstream supercritical flow appears. However, up to now, solutions for a forced Korteweg-de Vries equation with a negative forcing have not been completely studied [5]. In this paper $F$ is assumed to be near the critical value 1 , that is $F=1+\varepsilon \lambda$ and the same forced Korteweg-de Vires equation derived in [6] is used as our model equation, but we assume that the obstruction is negative and finite, and generates a negative forcing in the forced Korteweg-de Vires equation. Two cutoff values $\lambda_{1}, \lambda_{2}$ of the Froude number, where $0<\lambda_{1}<\lambda_{2}$, are found. Two positive symmetric solitary-wave-like solutions appear for $\lambda>\lambda_{1}$ and four positive symmetric or unsymmetric solitary-wave-like solutions appear for $\lambda>\lambda_{2}$. At the discrete values of positive $\lambda$ 's, another type of solitary-wave-like solution, which is zero ahead of the depression and a part of a solitary wave behind the depression, is also discovered. We also find positive symmetric solutions for discrete values of $\lambda<0$ and a negative solitarywave-like solution for $\lambda>0$.

Received 16th October, 2001

The research reported here is supported by Korea Research Foundation Grant (KRF-2000-DP0041).

Copyright Clearance Centre, Inc. Serial-fee code: 0004-9727/02 \$A2.00+0.00. 


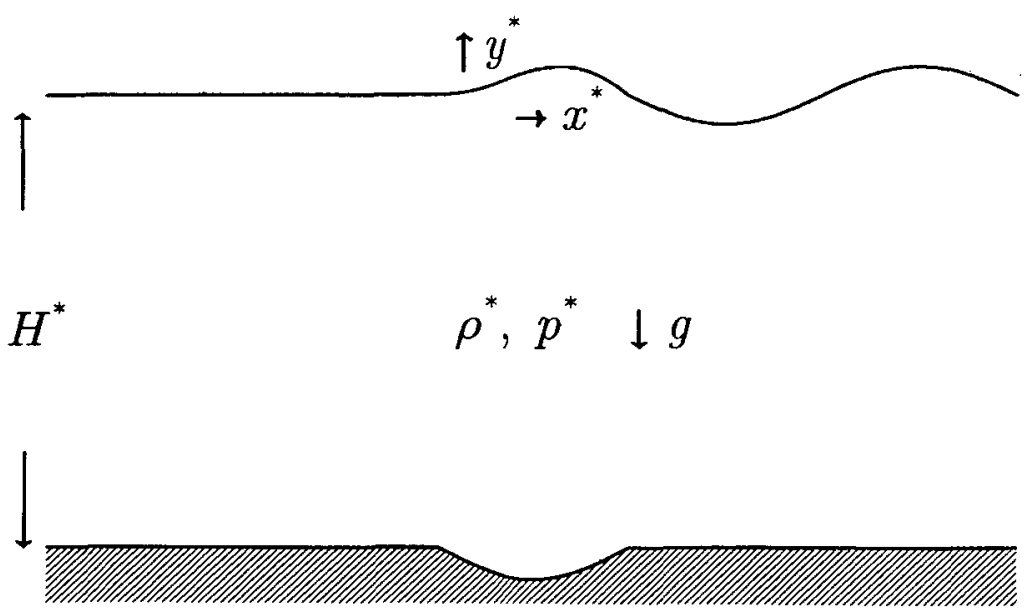

Figure 1: Fluid Domain

\section{Formulation AND NUmerical Results}

The problem considered here concerns steady two dimensional interfacial waves of a fluid with constant density passing over a depression with compact support (Figure 1). The governing equations and boundary conditions are as follows:

$$
\begin{aligned}
u_{x^{*}}^{*}+v_{y^{*}}^{*} & =0, \\
u^{*} u_{x^{*}}^{*}+v^{*} u_{y^{*}}^{*} & =p_{x^{*}}^{*} / \rho^{*}, \\
u^{*} v_{x^{*}}^{*}+v^{*} u v_{y^{*}}^{*} & =p_{y^{*}}^{*} / \rho^{*}-g,
\end{aligned}
$$

at the free surface $y^{*}=\eta^{*}\left(x^{*}\right)$,

$$
u^{*} \eta *_{x^{*}}-v^{*}=0, p^{*}=0,
$$

at the rigid lower boundary $y^{*}=h^{*}\left(x^{*}\right)$,

$$
v^{*}-u^{*} h_{x}^{*}=0,
$$

where $\left(u^{*}, v^{*}\right)$ are velocities, $p^{*}$ is the constant density of the fluid, $g$ is the gravitational acceleration constant, and $h^{*}\left(x^{*}\right)=-H^{*}+b^{*}\left(x^{*}\right)$, where $H^{*}$ is the constant depth of the fluid at equilibrium state, and $b^{*}\left(x^{*}\right)$ stands for the obstruction with finite support on the rigid bottom.

We define the following nondimensional variables:

$$
\begin{gathered}
\varepsilon=\left(H^{*} / L\right)^{1 / 2}, \eta=\varepsilon^{-1} \eta^{*} / H^{*}, x=\varepsilon^{1 / 2} x^{*} / H^{*}, y=y^{*} / H^{*}, p=p^{*} / g H^{*} \rho^{*}, \\
(u, v)=\left(g H^{*}\right)^{-1 / 2}\left(u^{*}, \varepsilon^{-1} v^{*}\right), h(x)=h^{*}(x) / H^{*}, b(x)=b(x)\left(H^{*} \varepsilon^{2}\right)^{-1},
\end{gathered}
$$


where $L$ is the horizontal length scale.

In the term of the above nondimensional variables and by assumimg that $u, v, p$ and $\eta$ possess an asymptotic expansion of the form

$$
\dot{\phi}=\phi_{0}+\varepsilon \phi_{1}+\varepsilon^{2} \phi_{2}+\cdots
$$

with $u_{0}=1, v_{0}=0, p_{0}=-y+1$, and the upstream Froude number $F=C /\left(g H^{*}\right)^{1 / 2}$ $=1+\varepsilon \lambda$, a system of differential equations and boundary conditions for successive approximations are obtained according to the order of $\varepsilon$. Then, by solving the resulting equation with the assumption $\eta(-\infty)=\eta_{1 x}(-\infty)=0$, we can derive the following forced Korteweg-de Vires equation (7),

$$
-\frac{1}{3} \eta_{1 x x x}-3 \eta_{1} \eta_{1 x}+2 \lambda \eta_{1 x}=b_{x}
$$

Integrating $(6)$ from $-\infty$ to $x$ yields

$$
\eta_{1 x x}=-\frac{9}{2} \eta_{1}^{2}+6 \lambda \eta_{1}-3 b(x)
$$

When $b(x)=0$ and $\lambda>0$, equation (7) can be solved directly and two solutions which vanish as $x$ tends to $\pm \infty$ are given as follows:

$$
\begin{aligned}
& \eta_{1}=2 \lambda \operatorname{sech}^{2}\left((6 \lambda)^{1 / 2}(x-\delta) / 2\right), \\
& \eta_{1}=-2 \lambda \operatorname{cosech}^{2}\left((6 \lambda)^{1 / 2}(x-\gamma) / 2\right),
\end{aligned}
$$

where $\delta$ and $\gamma$ are phase shifts. Equation (8) is the well-known solitary wave solution. Equation (9) is unbounded and has a singularity at $\gamma$. In what follows we shall call (9) an unbounded solitary wave solution.

Next we shall find a periodic solution of (7) without forcing. Assume $b(x)=0$ and $\eta_{1}$ and $\eta_{1 x}$ are given at some point $x=x_{0}$. Let $\eta_{1}\left(x_{0}\right)=\alpha$ and $\eta_{1 x}\left(x_{0}\right)=\beta$. Multiplying $\eta_{1 x}$ to $(7)$ with $b(x)=0$ and integrating the resulting equation from $x_{0}$ to $x>x_{0}$, we have

$$
\left(\eta_{1 x}\right)^{2}=-3 \eta_{1}^{3}+6 \lambda \eta_{1}^{2}+d=f\left(\eta_{1}\right)
$$

where $d=\beta^{2}+3 \alpha^{3}-6 \lambda \alpha^{2}$. Let $c_{1}, c_{2}$ and $c_{3}$ be three roots of $f\left(\eta_{1}\right)$. If all $c_{1}, c_{2}$ and $c_{3}$ are real and assume $c_{1}<c_{2}<c_{3}$ then (10) has the following periodic solution,

$$
\eta_{1}=c_{2}+\left(c_{3}-c_{2}\right) \operatorname{Sn}^{2}(\sqrt{3} M(x-\delta), k)
$$

where $k^{2}=\left(c_{3}-c_{2}\right) /\left(c_{3}-c_{1}\right), M^{2}=\left(c_{3}-c_{1}\right) / 4, \delta$ is a phase shift and $\mathrm{Sn}$ is the Jacobian Elliptic Function. As $c_{2} \uparrow c_{3}$, (11) tends to a constant solution $\eta_{1}=c_{2}$ and as $c_{1} \uparrow c_{2}$, (11) tends to the following non-periodic solution,

$$
\eta_{1}=c_{2}+\left(c_{3}-c_{2}\right) \operatorname{sech}^{2}\left(\left(3\left(c_{3}-c_{2}\right)\right)^{1 / 2}(x-\delta) / 2\right)
$$


Here $\delta$ is also a phase shift. In particular, if $c_{2}=0$ so that $d=0$ in (10), then (12) becomes (8). If two $c_{i}$ 's, $i=1,2,3$ are not real, the solution of (10) diverges.

In the following we shall assume $b(x)$ has a compact support $[-1,1]$ and consider two cases, $\lambda>0$ and $\lambda<0$.

CASE 1. Supercritical Case $\lambda>0$.

Since $\eta_{1}(-\infty)=0$ is assumed, only two types of solutions, $\eta_{1}=0$ and either bounded or unbounded solitary wave solutions, can appear for $x<-1$. We assume $b(x)=-\left(1-x^{2}\right)^{1 / 2}$ for $|x| \leqslant 1$ and $b(x)=0$ for $|x| \geqslant 1$ for the numerical solutions for (7) to follow. We consider the case of $\eta_{1}=0$ for $x \leqslant-1$ and the case of solitary waves and unbonded waves for $x \leqslant-1$ separately.

1. Solitary waves for $x \leqslant-1$.

We first construct positive solitary-wave-like solution numerically. Let

$$
\eta_{1}=2 \lambda \operatorname{sech}^{2}\left((6 \lambda)^{1 / 2}\left(x-\delta_{1}\right) / 2\right),
$$

for $x \leqslant-1$, and

$$
\eta_{1}=2 \lambda \operatorname{sech}^{2}\left((6 \lambda)^{1 / 2}\left(x-\delta_{2}\right) / 2\right),
$$

for $x \geqslant 1$, where $\delta_{1}$ and $\delta_{2}$ are phase shifts. To find a solution in $|x|<1$, we use a shooting method and the phase shifts $\delta_{1}$ and $\delta_{2}$ are determined by (13) and (14) for $x \leqslant-1$ and $x \geqslant 1$ respectively.

Negative solitary-wave-like solutions are numerically constructed by a similar method for the case of positive solitary-wave-like solution. We use a shooting method for (15)

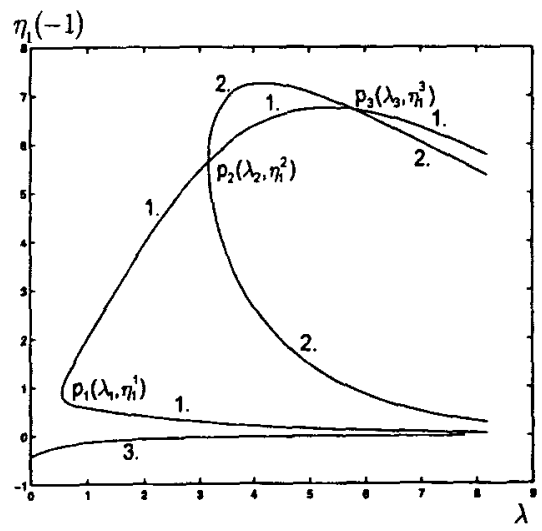

Figure 2: The relation between $\lambda$ and $\eta_{1}(-1)$, $\lambda_{1}=0.55192, \lambda_{2}=3.16415$. 1. Positive symmetric solitary-wave-like solution. 2. Postive unsymmetric solitary-wave-like solution. 3. Negative solitary-wave-like solution.

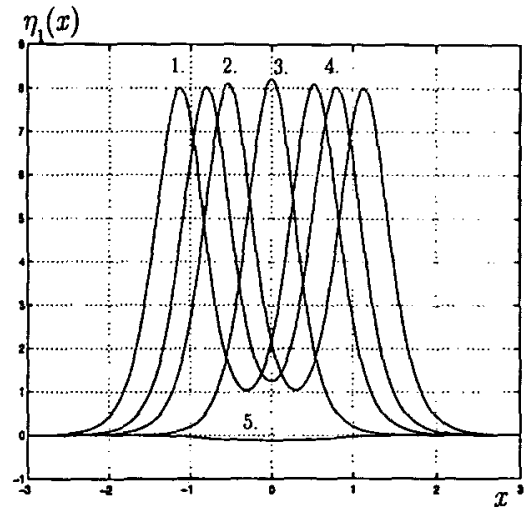

Figure 3: Typical solitary-wave-like solutions, $\lambda=4$. 1. and 2. Unsymmetric positive solitarywave-like solution. 3 . and 4 . Symmetric positive solitary-wave-like solution. 5. Symmetric negative solitary-wave-like solution. 
and (16) as follows:

$$
\begin{aligned}
& \eta_{1}=-2 \lambda \operatorname{cosech}^{2}\left((6 \lambda)^{1 / 2}\left(x-\gamma_{1}\right) / 2\right), \text { for } x \leqslant-1, \\
& \eta_{1}=-2 \lambda \operatorname{cosech}^{2}\left((6 \lambda)^{1 / 2}\left(x-\gamma_{2}\right) / 2\right), \text { for } x \geqslant-1,
\end{aligned}
$$

where $\gamma_{i}, i=1,2$ are also phase shifts.

The numerical results are given in Figures 2 and 3. The relation between $\lambda$ and $\eta_{1}(-1)$ for solitary-wave-like solutions is given in Figure 2. Two critical points $P_{1}\left(\lambda_{1}, \eta_{1}^{1}\right)$ and $P_{2}\left(\lambda_{2}, \eta_{2}^{1}\right)$ are given in Figure 2. For $\lambda>\lambda_{1}$, two positive symmetric solitary-wave-like solutions appear and, for $\lambda>\lambda_{2}$, two positive unsymmetric solitary-wave-like solutions appear. We note that the $\eta_{1 x}\left(P_{3}\right)$ corresponding to a positive symmetric solitary-wavelike solution is positive and the $\lambda>\lambda_{1}, \eta_{1 x}\left(P_{3}\right)$ corresponding to a positive unsymmetric solitary-wave-like solution is negative. Hence, two positive symmetric solitary-wave-like solutions and two positive unsymmetric solitary-wave-like solutions appear at $\lambda=\lambda_{3}$. A negative symmetric solitary-wave-like solution appears for any positive value of $\lambda$. We also note that negative symmetric solitary-wave-like solution and the cut-off point for the appearance of positive unsymmetric solitary-wave-like solutions do not occur if $b(x)$ is of the positive semicircular form [7]. Figure 3 shows two positive symmetric solitary-wavelike solutions, two unsymmetirc solitary-wave-like solutions, and one negative symmetric solitary-wave-like solution when $\lambda=4$. since we have derived the possible solutions of (7) in $[1, \infty)$ for any value of $\eta_{1}(1)$ and $\eta_{1 x}(1)$, we can solve (7) by Runge-Kutta Method using (13) for $(-\infty,-1]$. We present a typical periodic wave solution of this case in Figure 4.

(2) $\eta_{1}=0$ for $x \leqslant-1$.

We assume $\eta_{1}=0$ for $x \leqslant-1$ and solve (7) numerically. The numerical results are given in Figures 5 to 7 . Figure 5 shows a typical periodic wave and Figures 5 and 7 show the two unsymmetric solitary-wave-like solutions for critical values of $\lambda^{\prime} \mathrm{s}$. The solutions $\eta_{1}$ in Figure 6 and Figure 7 are 0 for $x \leqslant-1$ and determined by (8) for $x>1$.
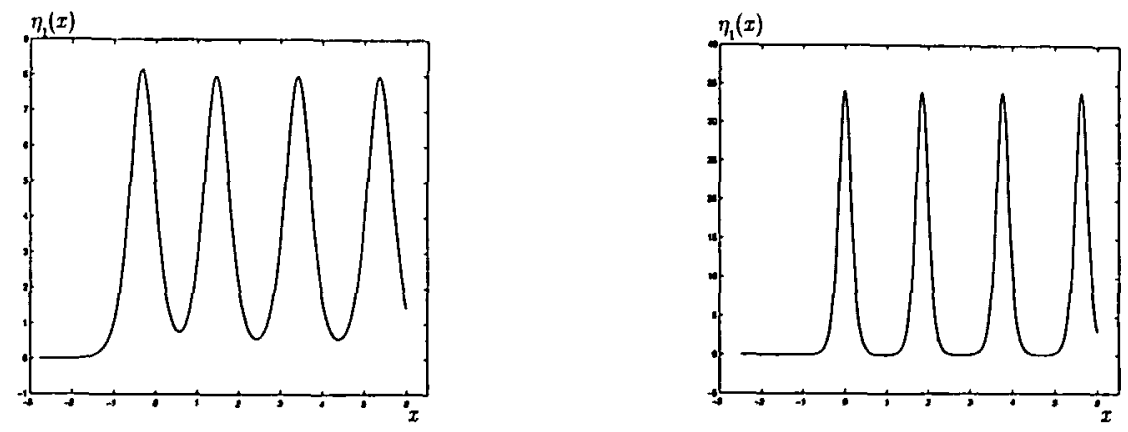

Figure 4: Typical periodic wave solution, $\lambda=4, \quad$ Figure 5: Typical periodic wave solution, $\eta_{1}=0$ for $\eta_{1}(-1)=1$. $x \leqslant-1, \lambda=17$. 


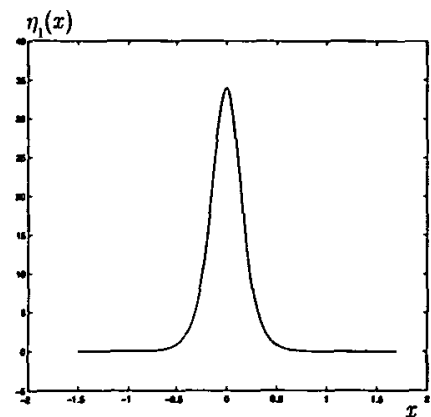

Figure 6: Unsymmetric solitary-wave-like solution, $\eta_{1}=0$ for $x \leqslant-1, \lambda=16.961718125$

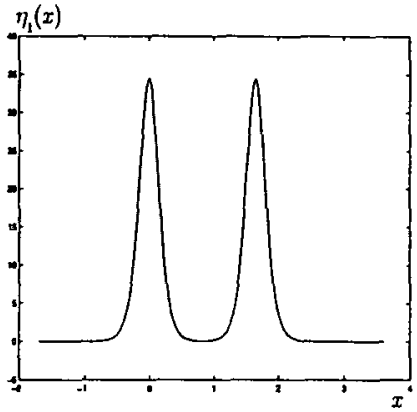

Figure 7: Unsymmetric solitary-wave-like solution, $\eta_{1}=0$ for $x \leqslant-1, \lambda=17.2243026835$

For $0<\lambda<16.9617$ and $\lambda>17.2243$,only unbounded solutions appear and periodic solutions appear for $16.9617<\lambda<17.2243$. We note that the two unsymmetric solitarywave-like solutions are the limiting cases of the periodic solutions.

CAsE 2. Subcritical Case $\lambda<0$.

In this caes, only $\eta_{1}=0$ can appear for $x<-1$ since we assumed $\eta_{1}(-\infty)=0$. We solve (7) by Runge-Kutta Method again. The numerical results of this case are given in Figure 8 to 11. In Figure 8, we present a hydraulic fall solution which is a limiting solution of periodic solutions. This solution appears at $\lambda=-0.79169272=\lambda_{s}$ and the solution diverges for $\lambda>\lambda_{s}$. We show a typical periodic solution in Figure 9. As $\lambda$ decreases, symmetric one hump solution appears as another type a limiting solution of periodic solutions and is shown in Figure 10. Multi-hump solutions take place for discrete value of $\lambda^{\prime}$ s. We present a symmetric two humps solution in Figure 11.
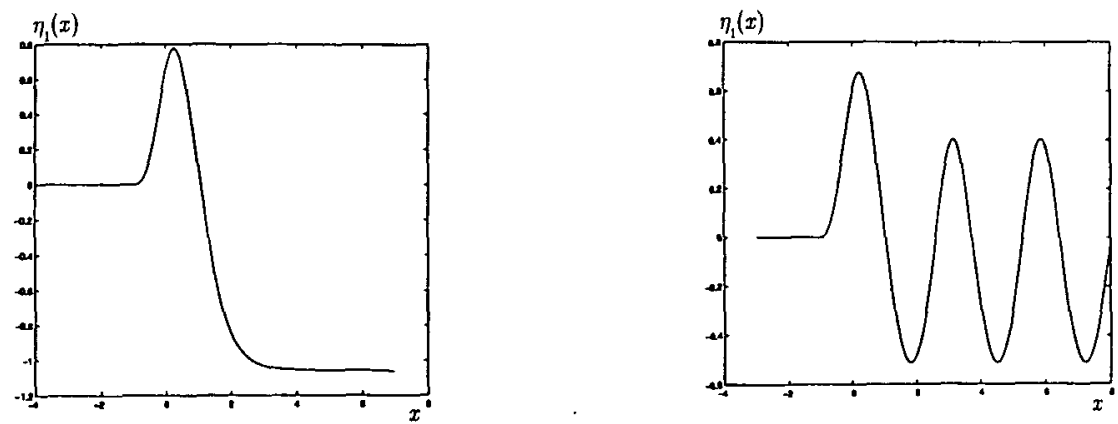

Figure 8: Hydrauric fall solution, $\eta_{1}=0$ for $x \leqslant-1, \quad$ Figure 9: Typical periodic wave solution, $\eta_{1}=0$ for $\lambda=-0.79169267$. $x \leqslant-1, \lambda=-1$. 


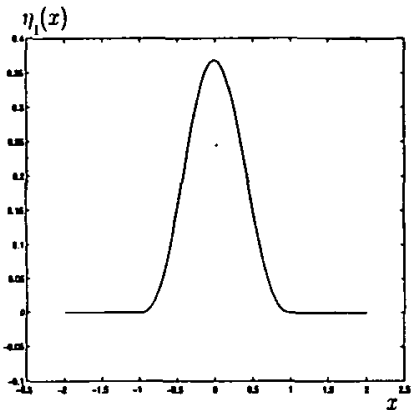

Figure 10: Symmetric wave solution with one hump, $\eta_{1}(-1)=0$ for $x \leqslant-1, \lambda=-2.14430583$.

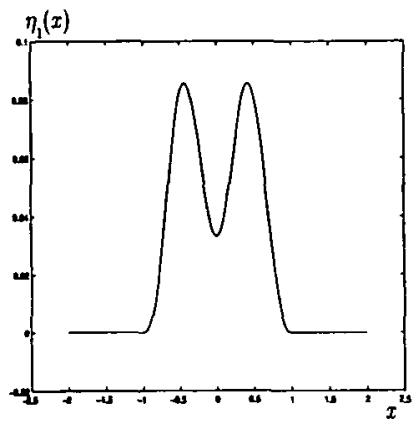

Figure 11: Symmetric wave solution with one humps, $\eta_{1}(-1)=0$ for $x \leqslant-1, \lambda=-6.1258501$.

\section{REFERENCES}

[1] S.L. Cole, 'Transient waves produced by flow past a bump', Wave Motion 7 (1985), 579-587.

[2] L.K. Forbes, 'Two-layer critical flow over a semi-circular obstruction', J. Eng. Math. 23 (1989), 325-342.

[3] L.K. Forbes and L.W. Schwartz, 'Free surface flow over a semi-circular obstruction', J. Fluid Mech. 144 (1982), 299-314.

[4] L. Gong and S.P. Shen, 'Multiple supercritical solitary wave solutions of the stationary forced Dorteweg-de Vires equation and their stability', SIAM J. Appl. Math. 54 (1994), $1268-1290$.

[5] S.P. Shen, 'Forced solitary waves and hydraulic falls in two layer flows', J. Fluid Mech. 234 (1992), 583-612.

[6] S.P. Shen and M.C. Shen, 'On the limit of subcritical free surface flow over an obstruction', Acta Mech. 82 (1990), 225-230.

[7] S.P. Shen, M.C. Shen and S.M. Sun, 'A model equation for steady surface wave over a bump', J. Engrg. Math. 23 (1989), 315-323.

[8] J.M. Vanden-Broeck, 'Free-surface flow over an obstruction in a channel', Phys. Fluids 30 (1987), 2315-2317.

[9] D.M. Wu and T.Y.Wu, 'Three-dimensional nonlinear long waves due to moving surface pressure', in Prac.14th Symp. Naval Hydrodyn. (National Academy of Science, Washington, 1982), pp. 103-125.

Department of Mathematics

Korea University

Seoul, 136-701

Korea

e-mail: jchoi@mail.korea.ac.kr 